J. Appl. Numer. Optim. 2 (2020), No. 2, pp. 187-197

Available online at http://jano.biemdas.com

https://doi.org/10.23952/jano.2.2020.2.05

\title{
WEAK CONVERGENCE OF A PRIMAL-DUAL ALGORITHM FOR SPLIT COMMON FIXED-POINT PROBLEMS IN HILBERT SPACES
}

\author{
DINGFANG HOU, JING ZHAO*, XINGLONG WANG \\ College of Science, Civil Aviation University of China, Tianjin 300300, China
}

\begin{abstract}
In this paper, we use the dual variable to propose a new iterative algorithm for solving the split common fixed-point problem of quasi-nonexpansive mappings in real Hilbert spaces. Under suitable conditions, we establish a weak convergence theorem of the proposed algorithm and obtain a related result for the split common fixed-point problem of firmly quasi-nonexpansive mappings. Some numerical experiments are given to illustrate the efficiency of the proposed iterative algorithm.
\end{abstract}

Keywords. Iterative algorithm; Quasi-nonexpansive mapping; Split common fixed-point problem; Weak convergence.

\section{INTRODUCTION}

Let $H_{1}$ and $H_{2}$ be two real Hilbert spaces. The problem under consideration in this paper is formulated as finding

$$
x^{*} \in F(U) \text { such that } A x^{*} \in F(T),
$$

where $A: H_{1} \rightarrow H_{2}$ is a bounded linear operator, $F(U)$ and $F(T)$ stand for the fixed point sets of $U: H_{1} \rightarrow H_{1}$ and $T: H_{2} \rightarrow H_{2}$, respectively. Problem (1.1) is called the split common fixed point problem (shortly, SCFP), which was introduced by Censor and Segal [5] in finite dimensional Hilbert spaces. In recent years, there has been growing interest in the SCFP due to its applications in the inverse problem of intensity-modulated radiation therapy and in the dynamic emission tomographic image reconstruction $[2,6,10]$.

In particular, if $U$ and $T$ are projection operators, then the SCFP is reduced to the well-known split feasibility problem (SFP) [2, 7], which consists in finding

$$
x^{*} \in C \text { such that } A x^{*} \in Q,
$$

where $C$ and $Q$ are nonempty closed convex subsets of $H_{1}$ and $H_{2}$, respectively. Such problems arise in the field of intensity-modulated radiation therapy when one attempts to describe physical dose constraints and equivalent uniform dose constraints within a single model (see [6]). Since the SFP in finite dimensional Hilbert spaces is introduced first by Censor and Elfving [7], many algorithms have been proposed to solve the SFP (see [3, 4, 8, 13, 14, 16, 18, 21] and references therein).

\footnotetext{
${ }^{*}$ Corresponding author.

E-mail addresses: 503156937@qq.com (D. Hou), zhaojing200103@163.com (J. Zhao), xinglong1979@163. com (X. Wang).

Received October 20, 2019; Accepted May 11, 2020.
}

(C)2020 Journal of Applied and Numerical Optimization 
For solving the SFP (1.2), Censor and Elfving [7] used multidistance method to obtain iterative algorithms, which involved matrix inverses at each step. In order to avoid usage of the inverse, Byrne [2] proposed an iterative method called CQ algorithm that involves only the orthogonal projections onto $C$ and $Q$. The CQ algorithm is defined as follows:

$$
x_{k+1}=P_{C}\left(I-\gamma A^{*}\left(I-P_{Q}\right) A\right) x_{k}
$$

for each $k \in \mathbb{N}$, where $P_{C}$ and $P_{Q}$ are the (orthogonal) projection onto $C$ and $Q$, respectively, and $\gamma \in\left(0, \frac{2}{\lambda}\right)$ with $\lambda$ being the spectral radius of the operator $A^{*} A$.

Note that, if split feasibility problem (1.2) is consistent (i.e., (1.2) has a solution), then it is no hard to see that $x^{*}$ solves the SFP (1.2) if and only if it solves the fixed point equation:

$$
P_{C}\left(I-\gamma A^{*}\left(I-P_{Q}\right) A\right) x^{*}=x^{*},
$$

where $\gamma>0$ and $A^{*}$ denotes the adjoint of $A$. This implies that we can use fixed point algorithms (see $[1,15,19,22,23]$ ) to solve the SFP.

For solving the SCFP (1.1) of directed operators (i.e. firmly quasi-nonexpansive mapping), Censor and Segal [5] proposed and proved, in finite dimensional spaces, the convergence of the following iterative scheme:

$$
x_{k+1}=U\left(x_{k}-\gamma A^{*}(I-T) A x_{k}\right),
$$

where $\gamma$ is chosen in the interval $\left(0, \frac{2}{\lambda}\right)$ with $\lambda$ being the spectral radius of the operator $A^{t} A\left(A^{t}\right.$ stands for matrix transposition). Many authors have introduced various algorithms to solve the $\operatorname{SCFP}(1.1)$ (see $[9,12,17,20,24,25])$.

For solving the SCFP (1.1) of quasi-nonexpansive mappings, Moudafi [17] introduced the following relaxed algorithm:

$$
x_{k+1}=\left(1-\alpha_{k}\right) u_{k}+\alpha_{k} U\left(u_{k}\right), \quad k \in N,
$$

where $u_{k}=x_{k}+\gamma \beta A^{*}(T-I) A x_{k}, \beta \in(0,1), \alpha_{k} \in(0,1)$ and $\gamma \in\left(0, \frac{1}{\eta \beta}\right)$ with $\eta$ being the spectral radius of the operator $A^{*} A$. Moudafi proved the weak convergence of the algorithm in Hilbert spaces.

In [11], Chen, Huang and Zhang considered to minimize the sum of two proper lower semicontinuous convex functions, i.e.,

$$
x^{*}=\arg \min _{x \in \mathbb{R}^{n}} f_{1}(x)+f_{2}(x),
$$

where $f_{1}, f_{2} \in \Gamma_{0}\left(\mathbb{R}^{n}\right)$ (all proper lower semi-continuous convex functions from $\mathbb{R}^{n}$ to $(-\infty,+\infty]$ ) and $f_{2}$ is differentiable on $\mathbb{R}^{n}$ with $1 / \beta$-Lipschitz continuous gradient for some $\beta \in(0,+\infty)$. To solve convex separable problem (1.6), they obtained the following fixed point formulation: the point $x^{*}$ is a solution of (1.6) if and only if there exists $v^{*} \in \mathbb{R}^{m}$ such that

$$
\left\{\begin{array}{l}
v^{*}=\left(I-\operatorname{prox}_{\frac{\gamma}{\lambda} f_{1}}\right)\left(x^{*}-\gamma \nabla f_{2}\left(x^{*}\right)+(1-\lambda) v^{*}\right), \\
x^{*}=x^{*}-\gamma \nabla f_{2}(x)-\lambda v^{*},
\end{array}\right.
$$

where $\lambda$ and $\gamma$ are two positive numbers. They introduced the following Picard iterative sequence:

$$
\left\{\begin{array}{l}
v_{k+1}=\left(I-\operatorname{prox}_{\frac{\gamma}{\lambda} f_{1}}\right)\left(x_{k}-\gamma \nabla f_{2}\left(x_{k}\right)+(1-\lambda) v_{k}\right) \\
x_{k+1}=x_{k}-\gamma \nabla f_{2}\left(x_{k}\right)-\lambda v_{k+1} .
\end{array}\right.
$$


It was shown [11] that, under appropriate conditions, the sequence $\left\{x_{k}\right\}$ converges to a solution of problem (1.6). Since $x$ is the primal variable related to (1.6), it is very natural to ask what role the variable $v$ plays in above algorithm. Indeed, $v$ is actually the dual variable of the primal-dual form related to (1.6).

Inspired and motivated by the works mentioned above, we use the dual variable to propose a new iterative algorithm for the SCFP governed by quasi-nonexpansive mappings. The organization of this paper is as follows. Some useful definitions and results are listed for the convergence analysis of the proposed iterative algorithms in Section 2. In Section 3, we introduce new iterative algorithm by the primal-dual method and the weak convergence theorem of the proposed iterative algorithm is obtained. We give a corollary for the SCFP (1.1) governed by firmly quasi-nonexpansive mappings. In Section 4, we also give some numerical experiments to illustrate the efficiency of the proposed iterative method.

\section{PRELIMINARIES}

In this paper, we denote the inner product by $\langle\cdot, \cdot\rangle$ and the norm by $\|\cdot\|$. We use $\rightarrow$ and $\rightarrow$ to denote the strong convergence and weak convergence, respectively. We use $\omega_{w}\left(x_{k}\right)=\{x$ : $\left.\exists x_{k_{j}} \rightarrow x\right\}$ to stand for the weak $\omega$-limit set of $\left\{x_{k}\right\}$. We use $\rightarrow$ and $\rightarrow$ to denote the strong convergence and weak convergence, respectively.

Definition 2.1. A mapping $T: H \rightarrow H$ is said to be

(i) nonexpansive if

$$
\|T x-T y\| \leq\|x-y\|
$$

for all $x, y \in H$;

(ii) firmly nonexpansive if $2 T-I$ is nonexpansive or, equivalently,

$$
\langle x-y, T x-T y\rangle \geq\|T x-T y\|^{2}
$$

for all $x, y \in H$. Alternatively, a mapping $T: H \rightarrow H$ is firmly nonexpansive if and only if $T$ can be expressed as

$$
T=\frac{1}{2}(I+S)
$$

where $I$ denotes the identity mapping on $H$ and $S: H \rightarrow H$ is a nonexpansive mapping;

(iii) quasi-nonexpansive if $F(T) \neq \emptyset$ and

$$
\|T x-q\| \leq\|x-q\|
$$

for all $x \in H$ and $q \in F(T)$;

(iv) firmly quasi-nonexpansive (also called directed operator) if $F(T) \neq \emptyset$ and

$$
\|T x-q\|^{2} \leq\|x-q\|^{2}-\|x-T x\|^{2}
$$

for all $x \in H$ and $q \in F(T)$.

Definition 2.2. A mapping $T: H \rightarrow H$ is said to be demiclosed at the origin if, for any sequence $\left\{x_{n}\right\}$ which converges weakly to $x$, the sequence $\left\{T x_{n}\right\}$ converges strongly to 0 , then $T x=0$. 
Lemma 2.1. [17] Let $H$ be a real Hilbert space, and let $T: H \rightarrow H$ be a quasi-nonexpansive mapping. Set $T_{\alpha}=(1-\alpha) I+\alpha T$ for $\alpha \in[0,1)$. Then the following properties are reached, for all $(x, q) \in H \times F(T)$,

(i) $\langle x-T x, x-q\rangle \geq \frac{1}{2}\|x-T x\|^{2}$ and $\langle x-T x, q-T x\rangle \leq \frac{1}{2}\|x-T x\|^{2}$;

(ii) $\left\|T_{\alpha} x-q\right\|^{2} \leq\|x-q\|^{2}-\alpha(1-\alpha)\|T x-x\|^{2}$;

(iii) $\left\langle x-T_{\alpha} x, x-q\right\rangle \geq \frac{\alpha}{2}\|x-T x\|^{2}$.

Remark 2.1. If $T_{\alpha}=(1-\alpha) I+\alpha T$, where $T: H \rightarrow H$ is a quasi-nonexpansive mapping and $\alpha \in(0,1)$, then $F\left(T_{\alpha}\right)=F(T)$ and $\left\|T_{\alpha} x-x\right\|^{2}=\alpha^{2}\|T x-x\|^{2}$. It follows from (ii) of Lemma 2.1 that $\left\|T_{\alpha} x-q\right\|^{2} \leq\|x-q\|^{2}-\frac{1-\alpha}{\alpha}\left\|T_{\alpha} x-x\right\|^{2}$, which implies that $T_{\alpha}$ is firmly quasinonexpansive when $\alpha=\frac{1}{2}$. On the other hand, if $\hat{T}$ is a firmly quasi-nonexpansive mapping, we can easily obtain $\hat{T}=\frac{1}{2} I+\frac{1}{2} T$, where $T$ is quasi-nonexpansive.

It follows from (iii) of Lemma 2.1 that the following result is easily obtained.

Proposition 2.1. Let $T$ be a quasi-nonexpansive mapping and $\alpha \in[0,1)$. If $T_{\alpha}=(1-\alpha) I+\alpha T$, then

$$
\left\|\left(I-T_{\alpha}\right) x\right\|^{2} \leq 2 \alpha\left\langle x-q,\left(I-T_{\alpha}\right) x\right\rangle
$$

for all $(x, q) \in H \times F(T)$.

Lemma 2.2. [22] Let $K$ be a nonempty convex closed subset of real Hilbert space $H$. Let $\left\{x_{k}\right\}$ be a bounded sequence which satisfies the following properties:

(a) every weak limit point of $\left\{x_{k}\right\}$ lies in $K$;

(b) $\lim _{k \rightarrow \infty}\left\|x_{k}-x\right\|$ exists for every $x \in K$.

Then $\left\{x_{k}\right\}$ converges weakly to a point in $K$.

\section{WEAK CONVERGENCE RESULTS}

In this section, we assume that the SCFP (1.1) is always consistent and its solution set is denoted by $\Gamma$, i.e.,

$$
\Gamma=\{x \in F(U): A x \in F(T)\} .
$$

We always assume the $H_{1}$ and $H_{2}$ are two real Hilbert spaces and $A: H_{1} \rightarrow H_{2}$ is a bounded linear operator. Assume $U: H_{1} \rightarrow H_{1}$ and $T: H_{2} \rightarrow H_{2}$ be quasi-nonexpansive mappings and let $U_{\alpha_{1}}=\left(1-\alpha_{1}\right) I+\alpha_{1} U$ and $T_{\alpha_{2}}=\left(1-\alpha_{2}\right) I+\alpha_{2} T$, where $\alpha_{1}, \alpha_{2} \in(0,1)$.

Now, we use the dual variable to propose the new iterative algorithm for the SCFP (1.1) governed by quasi-nonexpansive mapping.

Algorithm 3.1. Let $x_{0}, v_{0} \in H_{1}$ be arbitrarily chosen, and $\lambda \in(0,1]$. For $k \geq 1$, let

$$
\left\{\begin{array}{l}
y_{k}=x_{k}-\gamma_{k} A^{*}\left(I-T_{\alpha_{2}}\right) A x_{k}, \\
v_{k+1}=\left(I-U_{\alpha_{1}}\right)\left(y_{k}+(1-\lambda) v_{k}\right), \\
x_{k+1}=y_{k}-\lambda v_{k+1}
\end{array}\right.
$$

where the stepsize $\gamma_{k}$ satisfies

$$
0<\liminf _{k \rightarrow \infty} \gamma_{k} \leq \limsup _{k \rightarrow \infty} \gamma_{k}<\frac{1}{\alpha_{2}\|A\|^{2}} .
$$


Theorem 3.1. Let $\alpha_{1} \in\left(0, \frac{1}{2}\right]$, and $I-U$ and $I-T$ be demiclosed at origin. Let $\left\{\left(v_{k}, x_{k}\right)\right\}$ be the sequence generated by Algorithm 3.1. Then $\left\{x_{k}\right\}$ converges weakly to a solution $x^{*} \in \Gamma$ and the sequence $\left\{\left(v_{k}, x_{k}\right)\right\}$ weakly converges to $\left(0, x^{*}\right)$.

Proof. First, we show that $\lim _{k \rightarrow \infty}\left\|x_{k}-x^{*}\right\|$ exists for any $x^{*} \in \Gamma$. Taking $x^{*} \in \Gamma$, we have $x^{*} \in F(U)$ and $A x^{*} \in F(T)$. By Algorithm 3.1 and Proposition 2.1, we have

$$
\begin{aligned}
\left\|v_{k+1}\right\|^{2} & =\left\|\left(I-U_{\alpha_{1}}\right)\left(y_{k}+(1-\lambda) v_{k}\right)\right\|^{2} \\
& =\left\|\left(I-U_{\alpha_{1}}\right)\left(y_{k}+(1-\lambda) v_{k}\right)-\left(I-U_{\alpha_{1}}\right) x^{*}\right\|^{2} \\
& \leq 2 \alpha_{1}\left\langle v_{k+1}, y_{k}-x^{*}+(1-\lambda) v_{k}\right\rangle
\end{aligned}
$$

and

$$
\begin{aligned}
\left\|x_{k+1}-x^{*}\right\|^{2} & =\left\|y_{k}-\lambda v_{k+1}-x^{*}\right\|^{2} \\
& =\left\|y_{k}-x^{*}\right\|^{2}-2 \lambda\left\langle y_{k}-x^{*}, v_{k+1}\right\rangle+\lambda^{2}\left\|v_{k+1}\right\|^{2} .
\end{aligned}
$$

It follows that

$$
\begin{aligned}
\left\|x_{k+1}-x^{*}\right\|^{2}+\lambda\left\|v_{k+1}\right\|^{2} \\
=\left\|y_{k}-x^{*}\right\|^{2}-2 \lambda\left\langle y_{k}-x^{*}, v_{k+1}\right\rangle+\lambda^{2}\left\|v_{k+1}\right\|^{2}+\lambda\left\|v_{k+1}\right\|^{2} \\
=\left\|y_{k}-x^{*}\right\|^{2}-2 \lambda\left\langle y_{k}-x^{*}, v_{k+1}\right\rangle+\frac{1}{\alpha_{1}} \lambda\left\|v_{k+1}\right\|^{2}-\lambda\left(\frac{1}{\alpha_{1}}-1-\lambda\right)\left\|v_{k+1}\right\|^{2} \\
\leq\left\|y_{k}-x^{*}\right\|^{2}-2 \lambda\left\langle y_{k}-x^{*}, v_{k+1}\right\rangle+2 \lambda\left\langle y_{k}-x^{*}+(1-\lambda) v_{k}, v_{k+1}\right\rangle \\
\quad-\lambda\left(\frac{1}{\alpha_{1}}-1-\lambda\right)\left\|v_{k+1}\right\|^{2} \\
=\left\|y_{k}-x^{*}\right\|^{2}+2 \lambda(1-\lambda)\left\langle v_{k}, v_{k+1}\right\rangle-\lambda\left(\frac{1}{\alpha_{1}}-1-\lambda\right)\left\|v_{k+1}\right\|^{2} .
\end{aligned}
$$

Observe that

$$
2\left\langle v_{k+1}, v_{k}\right\rangle=\left\|v_{k+1}\right\|^{2}+\left\|v_{k}\right\|^{2}-\left\|v_{k+1}-v_{k}\right\|^{2} .
$$

From (3.1) and (3.2), we obtain

$$
\begin{aligned}
\left\|x_{k+1}-x^{*}\right\|^{2}+\lambda\left\|v_{k+1}\right\|^{2} \leq & \left\|y_{k}-x^{*}\right\|^{2}+\lambda(1-\lambda)\left\|v_{k}\right\|^{2} \\
& -\lambda(1-\lambda)\left\|v_{k+1}-v_{k}\right\|^{2}-\lambda\left(\frac{1}{\alpha_{1}}-2\right)\left\|v_{k+1}\right\|^{2} .
\end{aligned}
$$

In view of

$$
\begin{aligned}
\left\langle x_{k}-x^{*}, A^{*}\left(I-T_{\alpha_{2}}\right) A x_{k}\right\rangle & =\left\langle A x_{k}-A x^{*},\left(I-T_{\alpha_{2}}\right) A x_{k}-\left(I-T_{\alpha_{2}}\right) A x^{*}\right\rangle \\
& \geq \frac{1}{2 \alpha_{2}}\left\|\left(I-T_{\alpha_{2}}\right) A x_{k}-\left(I-T_{\alpha_{2}}\right) A x^{*}\right\|^{2} \\
& =\frac{1}{2 \alpha_{2}}\left\|\left(I-T_{\alpha_{2}}\right) A x_{k}\right\|^{2},
\end{aligned}
$$


we have

$$
\begin{aligned}
\left\|y_{k}-x^{*}\right\|^{2} & =\left\|x_{k}-\gamma_{k} A^{*}\left(I-T_{\alpha_{2}}\right) A x_{k}-x^{*}\right\|^{2} \\
& \leq\left\|x_{k}-x^{*}\right\|^{2}-2 \gamma_{k}\left\langle x_{k}-x^{*}, A^{*}\left(I-T_{\alpha_{2}}\right) A x_{k}\right\rangle+\gamma_{k}^{2}\|A\|^{2}\left\|\left(I-T_{\alpha_{2}}\right) A x_{k}\right\|^{2} \\
& \leq\left\|x_{k}-x^{*}\right\|^{2}-\frac{1}{\alpha_{2}} \gamma_{k}\left\|\left(I-T_{\alpha_{2}}\right) A x_{k}\right\|^{2}+\gamma_{k}^{2}\|A\|^{2}\left\|\left(I-T_{\alpha_{2}}\right) A x_{k}\right\|^{2} \\
& =\left\|x_{k}-x^{*}\right\|^{2}-\gamma_{k}\left(\frac{1}{\alpha_{2}}-\gamma_{k}\|A\|^{2}\right)\left\|\left(I-T_{\alpha_{2}}\right) A x_{k}\right\|^{2} .
\end{aligned}
$$

It follows that

$$
\begin{aligned}
& \left\|x_{k+1}-x^{*}\right\|^{2}+\lambda\left\|v_{k+1}\right\|^{2} \\
& \leq\left\|x_{k}-x^{*}\right\|^{2}-\gamma_{k}\left(\frac{1}{\alpha_{2}}-\gamma_{k}\|A\|^{2}\right)\left\|\left(I-T_{\alpha_{2}}\right) A x_{k}\right\|^{2}+\lambda(1-\lambda)\left\|v_{k}\right\|^{2} \\
& \quad-\lambda(1-\lambda)\left\|v_{k+1}-v_{k}\right\|^{2}-\lambda\left(\frac{1}{\alpha_{1}}-2\right)\left\|v_{k+1}\right\|^{2} \\
& =\left\|x_{k}-x^{*}\right\|^{2}+\lambda\left\|v_{k}\right\|^{2}-\gamma_{k}\left(\frac{1}{\alpha_{2}}-\gamma_{k}\|A\|^{2}\right)\left\|\left(I-T_{\alpha_{2}}\right) A x_{k}\right\|^{2}-\lambda^{2}\left\|v_{k}\right\|^{2} \\
& \quad-\lambda(1-\lambda)\left\|v_{k+1}-v_{k}\right\|^{2}-\lambda\left(\frac{1}{\alpha_{1}}-2\right)\left\|v_{k+1}\right\|^{2} .
\end{aligned}
$$

Let

$$
s_{k}=\left\|x_{k}-x^{*}\right\|^{2}+\lambda\left\|v_{k}\right\|^{2} .
$$

By the assumptions on $\gamma_{k}, \lambda, \alpha_{1}, \alpha_{2}$ and (3.3), we obtain that $s_{k+1} \leq s_{k}$, which implies that sequence $\left\{s_{k}\right\}$ is non-increasing. Since $\left\{s_{k}\right\}$ is lower bounded by 0 , we have that $\lim _{k \rightarrow \infty} s_{k}$ exists. Thus it follows that $\left\{s_{k}\right\}$ is bounded. Hence $\left\{x_{k}\right\}$ is bounded.

Moreover, from (3.3), we also have

$$
\gamma_{k}\left(\frac{1}{\alpha_{2}}-\gamma_{k}\|A\|^{2}\right)\left\|\left(I-T_{\alpha_{2}}\right) A x_{k}\right\|^{2}+\lambda^{2}\left\|v_{k}\right\|^{2} \leq s_{k}-s_{k+1}
$$

which implies that

$$
\lim _{k \rightarrow \infty}\left\|\left(I-T_{\alpha_{2}}\right) A x_{k}\right\|=0
$$

and

$$
\lim _{k \rightarrow \infty}\left\|v_{k}\right\|=0 .
$$

So, we obtain that $\lim _{k \rightarrow \infty}\left\|x_{k}-x^{*}\right\|^{2}=\lim _{k \rightarrow \infty}\left(s_{k}-\lambda\left\|v_{k}\right\|^{2}\right)=\lim _{k \rightarrow \infty} s_{k}$ exists. In view of

$$
\left\|x_{k}-y_{k}\right\|=\left\|-\gamma_{k} A^{*}\left(I-T_{\alpha_{2}}\right) A x_{k}\right\|
$$

we obtain from (3.4) that $\lim _{k \rightarrow \infty}\left\|x_{k}-y_{k}\right\|=0$. This shows that

$$
\lim _{k \rightarrow \infty}\left\|x_{k}-\left(y_{k}+(1-\lambda) v_{k}\right)\right\|=0 \text {. }
$$

Next, we prove that $\omega_{w}\left(x_{k}\right) \subseteq \Gamma$. Taking $\bar{x} \in \omega_{w}\left(x_{k}\right)$, i.e., there exists a subsequence $\left\{x_{k_{j}}\right\}$ of $\left\{x_{k}\right\}$ such that $x_{k_{j}} \rightarrow \bar{x}$, we have $A x_{k_{j}} \rightarrow A \bar{x}$ as $j \rightarrow \infty$. Using (3.5), we have $y_{k_{j}}+(1-\lambda) v_{k_{j}} \rightarrow \bar{x}$ as $j \rightarrow \infty$. Moreover,

$$
\lim _{k \rightarrow \infty}\left\|v_{k+1}\right\|=\lim _{k \rightarrow \infty}\left\|\left(I-U_{\alpha_{1}}\right)\left(y_{k}+(1-\lambda) v_{k}\right)\right\|=0 .
$$


Note that $I-U$ and $I-T$ are demiclosed at 0 . From $I-U_{\alpha_{1}}=\left(1-\alpha_{1}\right)(I-U)$ and $I-T_{\alpha_{2}}=$ $\left(1-\alpha_{2}\right)(I-T)$, we have that $I-U_{\alpha_{1}}$ and $I-T_{\alpha_{2}}$ are also demiclosed at 0 . Using (3.4) and (3.6), we have $\bar{x} \in F\left(U_{\alpha_{1}}\right)=F(U)$ and $A \bar{x} \in F\left(T_{\alpha_{2}}\right)=F(T)$, which imply that $\bar{x} \in \Gamma$. So $\omega_{w}\left(x_{k}\right) \subseteq \Gamma$ is proved.

Finally, by using Lemma 2.2, we have $x_{k} \rightarrow x^{*}$, where $x^{*}$ is a solution of the SCFP (1.1). It follows from $v_{k} \rightarrow 0$ that $\left(v_{k}, x_{k}\right) \rightarrow\left(0, x^{*}\right)$. This completes the proof.

Remark 3.1. For the particular case, " $\lambda=1$ ", Algorithm 3.1 becomes the following iterative algorithm for solving the SCFP (1.1) of quasi-nonexpansive mappings:

$$
x_{k+1}=U_{\alpha_{1}}\left(x_{k}-\gamma_{k} A^{*}\left(I-T_{\alpha_{2}}\right) x_{k}\right),
$$

where $\alpha_{1} \in\left(0, \frac{1}{2}\right]$ and the stepsize $\gamma_{k}$ satisfies

$$
0<\liminf _{k \rightarrow \infty} \gamma_{k} \leq \limsup _{k \rightarrow \infty} \gamma_{k}<\frac{1}{\alpha_{2}\|A\|^{2}} .
$$

Algorithm 3.7 can be rewritten as

$$
x_{k+1}=\left(\left(1-\alpha_{1}\right) I+\alpha_{1} U\right)\left(x_{k}-\gamma_{k} \alpha_{2} A^{*}(I-T) x_{k}\right),
$$

which becomes algorithm (1.5) proposed by Moudafi [17] for solving the SCFP (1.1) of quasinonexpansive mappings.

We now turn our attention to the application of the proposed algorithm to the SCFP (1.1) governed by firmly quasi-nonexpansive mappings. Since any firmly quasi-nonexpansive mapping is quasi-nonexpansive, we can straightly obtain Algorithm 3.1 for solving the SCFP (1.1) of firmly quasi-nonexpansive mappings. From Remark 2.1, we know that any firmly quasinonexpansive mapping can be expressed be the $\frac{1}{2}$-relaxed operator of quasi-nonexpansive mapping. Algorithm 3.1 takes the following equivalent form for solving the SECFP (1.1) of firmly quasi-nonexpansive mappings $U$ and $T$.

Algorithm 3.2. Let $x_{0}, v_{0} \in H_{1}$ be arbitrarily chosen, and $\lambda \in(0,1]$. For $k \geq 1$, let

$$
\left\{\begin{array}{l}
y_{k}=x_{k}-\gamma_{k} A^{*}(I-T) A x_{k}, \\
v_{k+1}=(I-U)\left(y_{k}+(1-\lambda) v_{k}\right), \\
x_{k+1}=y_{k}-\lambda v_{k+1}
\end{array}\right.
$$

where the stepsize $\gamma_{k}$ satisfies

$$
0<\liminf _{k \rightarrow \infty} \gamma_{k} \leq \limsup _{k \rightarrow \infty} \gamma_{k}<\frac{2}{\|A\|^{2}}
$$

Corollary 3.1. Let $U: H_{1} \rightarrow H_{1}$ and $T: H_{2} \rightarrow H_{2}$ be firmly quasi-nonexpansive mappings. Assume that $I-U$ and $I-T$ are demiclosed at origin. Let the sequence $\left\{\left(v_{k}, x_{k}\right)\right\}$ be generated by Algorithm 3.2. Then the sequence $\left\{x_{k}\right\}$ converges weakly to a solution $x^{*} \in \Gamma$ and the sequence $\left\{\left(v_{k}, x_{k}\right)\right\}$ weakly converges to $\left(0, x^{*}\right)$.

Remark 3.2. For the particular case, " $\lambda=1$ ", Algorithm 3.2 becomes the following iterative algorithm for solving the SCFP (1.1) of firmly quasi-nonexpansive mappings:

$$
x_{k+1}=U\left(x_{k}-\gamma_{k} A^{*}(I-T) x_{k}\right),
$$


where the stepsize $\gamma_{k}$ satisfies

$$
0<\liminf _{k \rightarrow \infty} \gamma_{k} \leq \limsup _{k \rightarrow \infty} \gamma_{k}<\frac{2}{\|A\|^{2}}
$$

Algorithm (3.8) becomes algorithm (1.4) which was introduced by Censor and Segal [5] for solving the SCFP (1.1) of firmly quasi-nonexpansive mappings.

\section{NUMERICAL EXPERIMENTS}

In this section, we provide some numerical experiments and show the performance of the proposed primal-dual iterative Algorithm 3.1 for solving the SCFP (1.1). All the codes are written in MATLAB and are performed on a personal Lenovo computer with Intel(R) Core(TM)i76500U CPU @ 2.5GHz and RAM 8.00GB.

Example 4.1. Let $R^{2}$ be the two dimensional Euclidean space with inner product

$$
\langle x, y\rangle=x^{(1)} y^{(1)}+x^{(2)} y^{(2)}
$$

and the norm $\|x\|=\sqrt{\left(x^{(1)}\right)^{2}+\left(x^{(2)}\right)^{2}}$ for all $x=\left(x^{(1)}, x^{(2)}\right)^{T}, y=\left(y^{(1)}, y^{(2)}\right)^{T} \in R^{2}$. Defined $U: R^{2} \rightarrow R^{2}$ by

$$
U: x=\left(x^{(1)}, x^{(2)}\right)^{T} \mapsto\left(x^{(1)}, \sin x^{(2)}\right)^{T} .
$$

Obviously, $U$ is quasi-nonexpansive mapping and the set of fixed points of $U$, denoted by $F(U)=\left\{\left(x^{(1)}, 0\right) \mid x^{(1)} \in R\right\}$, is not empty. Let the nonempty closed convex set $Q_{1}=\{y=$ $\left.\left(y^{(1)}, y^{(2)}\right)^{T} \in R^{2} \mid 1 \leq y_{j} \leq 2, j=1,2\right\}$ and $Q_{2}=\left\{y \in R^{2} \mid\left\|y-(2,1.5)^{T}\right\| \leq 1\right\}$. Let the matrix

$$
A=\left(\begin{array}{cc}
2 & -1 \\
2 & 2
\end{array}\right) \text {. }
$$

We consider the following problem:

$$
\text { finding } x^{*} \in F(U) \text { such that } A x^{*} \in Q_{1} \bigcap Q_{2} \text {. }
$$

We know the metric projections onto the sets $Q_{1}$ and $Q_{2}$ have closed-form expressions. We can compute the projection onto the set $Q_{1}$ with

$$
P_{Q_{1}}(x)=\left\{z=\left(z^{(1)}, z^{(2)}\right)^{T} \mid z^{(i)}=\max \left\{1, \min \left\{x^{(i)}, 2\right\}\right\}, i=1,2\right\}, x=\left(x^{(1)}, x^{(2)}\right)^{T} \in R^{2} .
$$

Since the set $Q_{2}$ is a closed ball, the projection onto the set $Q_{2}$ can be computed with

$$
P_{Q_{2}}(x):= \begin{cases}c+\frac{r}{\|x-c\|^{2}}(x-c), & \|x-c\|>r \\ x, & \|x-c\| \leq r\end{cases}
$$

where $c=(2,1.5)^{T}$ and $r=1$. Taking $T=P_{Q_{2}} P_{Q_{1}}$, we have $T$ is quasi-nonexpansive mapping and

$$
F(T)=F\left(P_{Q_{2}}\right) \bigcap F\left(P_{Q_{1}}\right)=Q_{1} \bigcap Q_{2}
$$

where $F(T)$ is the nonempty set of fixed points of $T$. The problem (4.1) becomes the following SCFP:

$$
\text { finding } x^{*} \in F(U) \text { such that } A x^{*} \in F(T) \text {. }
$$

Now we turn to realizing primal-dual iterative Algorithm 3.1 for approximating a solution of problem (4.2) by using Theorem 3.1. We take $\alpha_{1}=\alpha_{2}=\frac{1}{2}$ and

$$
p_{k}=\left\|x_{k}-U x_{k}\right\|+\left\|A x_{k}-T A x_{k}\right\|<\varepsilon=10^{-5}
$$


TABLE 1. Numerical results for solving Example 4.1 with different $\lambda$.

\begin{tabular}{cccc}
\multicolumn{4}{c}{$x_{0}=(-5,-4)^{T}, v_{0}=(0,0)^{T}$} \\
\hline$\lambda$ & $k$ & $t$ & $p_{k}$ \\
0.1 & 13 & 0.0001 & $9.5973 \times 10^{-6}$ \\
0.2 & 13 & 0.0001 & $7.5569 \times 10^{-7}$ \\
0.6 & 12 & 0.0001 & $5.9308 \times 10^{-7}$ \\
1(algorithm (3.7)) & 2756 & 0.2500 & $9.9974 \times 10^{-6}$ \\
\hline
\end{tabular}

TABLE 2. Numerical results for solving Example 4.1 with different $\lambda$.

\begin{tabular}{cccc}
\multicolumn{4}{c}{$x_{0}=(-5,0)^{T}, v_{0}=(1,0)^{T}$} \\
\hline$\lambda$ & $k$ & $t$ & $p_{k}$ \\
0.6 & 10 & 0.0001 & $2.7678 \times 10^{-6}$ \\
0.7 & 10 & 0.0001 & $2.3466 \times 10^{-7}$ \\
0.8 & 1827 & 0.1719 & $9.9978 \times 10^{-6}$ \\
1(algorithm (3.7)) & 3581 & 0.2656 & $9.9981 \times 10^{-6}$ \\
\hline
\end{tabular}

TABLE 3. Numerical results for solving Example 4.1 with different $\lambda$.

\begin{tabular}{cccc}
\multicolumn{4}{c}{$x_{0}=(10,0)^{T}, v_{0}=(0,0)^{T}$} \\
\hline$\lambda$ & $k$ & $t$ & $p_{k}$ \\
0.3 & 14 & 0.0001 & $2.0679 \times 10^{-6}$ \\
0.6 & 13 & 0.0001 & $1.7704 \times 10^{-6}$ \\
0.9 & 2184 & 0.1406 & $9.9973 \times 10^{-6}$ \\
1(algorithm (3.7)) & 2682 & 0.2188 & $9.9973 \times 10^{-6}$ \\
\hline
\end{tabular}

as the stopping criterion. We take $x_{0}=\left(x_{0}^{(1)}, x_{0}^{(2)}\right)^{T}$ and $v_{0}=\left(v_{0}^{(1)}, v_{0}^{(2)}\right)^{T}$ as initial points.

In all the tables below, ' $k$ ' and ' $t$ 'denote the number of iterations and the total computing time in seconds. We take $\gamma_{k}=\frac{1.95}{\|A\|^{2}}$ and

$$
p_{k}=\left\|x_{k}-U x_{k}\right\|+\left\|A x_{k}-T A x_{k}\right\|
$$

as error estimation of our algorithm. We take different values of $\lambda, x_{0}$ and $v_{0}$ for solving this example in Table 1-3. When the parameter $\lambda=1$, the Algorithm 3.1 becomes algorithm (3.7) which was proposed by Moudafi [17] for solving the SCFP (1.1) of quasi-nonexpansive mappings. We take $x_{0}=(-5,-4), v_{0}=(0,0) ; x_{0}=(-5,0), v_{0}=(1,0) ; x_{0}=(10,0), v_{0}=(0,0)$ in numerical experiments. We compare our proposed Algorithm 3.1 with algorithm (3.7).

We can see from Table 1-3 that Algorithm 3.1 is efficient and behaved better than algorithm (3.7) if we choose a suitable parameter $\lambda$ for solving Example 4.1. So, the proper choice of the parameter $\lambda \in(0,1]$ may accelerate the convergence. 


\section{THE CONCLUSION}

In this paper, we may take parameter $\lambda \in(0,1]$. If $\lambda=1$, then Algorithm 3.1 is reduced to algorithm (3.7), which was proposed by Moudafi [17] for solving the SCFP (1.1) of quasinonexpansive mappings. Similarly, if $\lambda=1$, then Algorithm 3.1 is reduced to algorithm (3.8) (i.e. (1.4)), which was introduced by Censor and Segal [5] for solving the SCFP (1.1) of firmly quasi-nonexpansive mappings.

\section{Acknowledgements}

The authors are grateful the anonymous referee for useful comments which improved this paper. This paper was supported by the Scientific Research Project of Tianjin Municipal Education Commission (No. 2018KJ253).

\section{REFERENCES}

[1] H.H Bauschke, J.M Borwein, On projection algorithms for solving convex feasibility problems, SIAM Rev. 38 (1996), 367-426.

[2] C. Byrne, Iterative oblique projection onto convex subsets and the split feasibility problem, Inverse Probl. 18 (2002), 441-453.

[3] C. Byrne, A unified treatment of some iterative algorithms in signal processing and image reconstruction, Inverse Probl. 20 (2004), 103-120.

[4] C. Byrne, Y. Censor, A. Gibali, S. Reich, The split common null point problem, J. Nonlinear Convex Anal. 13 (2012), 759-775.

[5] Y. Censor, A. Segal, The split common fixed point problem for directed operators, J. Convex Anal. 16 (2009), 587-600.

[6] Y. Censor, T. Bortfeld, B. Martin, A. Trofimov, A unified approach for inversion problems in intensity modulated radiation therapy, Phys. Med. Biol. 51 (2006), 2353-2365.

[7] Y. Censor, T. Elfving, A multiprojection algorithm using Bregman projections in a product space, Numer. Algo. 8 (1994), 221-239.

[8] Y. Censor, A. Gibalin, S. Reich, Algorithms for the split variational inequality problem, Numer. Algo. 59 (2012), 301-323.

[9] A. Cegielski, General method for solving the split common fixed point problem, J. Optim. Theory Appl. 165 (2015), 385-404.

[10] P.L. Combettes, The convex feasibility problem in image recovery, Adv. Imaging Electron. Phys. 95 (1996), 155-270.

[11] P. Chen, J. Huang, X. Zhang, A primal-dual fixed point algorithm for convex separable minimization with applications to image restoration, Inverse Probl. 29 (2013), 025011.

[12] H. Cui, F. Wang, Iterative methods for the split commom fixed point problem in Hilbert spaces, Fixed Point Theory Appl. 2014 (2014), Article ID 78.

[13] Q.L. Dong, Y.C. Tang, Y.J. Cho, Th.M. Rassias, "Optimal" choice of the step length of the projection and contraction methods for solving the split feasibility problem, J. Glob. Optim. 71 (2018), 341-360.

[14] Q.L. Dong, H.B. Yuan, Y.J. Cho, Th.M. Rassias, Modified inertial Mann algorithm and Inertial CQ-algorithm for nonexpansive mappings, Optim. Lett. 12 (2018), 87-102.

[15] S. He, H. Tian, H.K. Xu, The selective projection method for convex feasibility and split feasibility problems, J. Nonlinear Convex Anal. 19 (2018), 1199-1215.

[16] E. Masad, S. Reich, A note on the multiple-set split convex feasibility problem in Hilbert space, J. Nonlinear Convex Anal. 8 (2007), 367-371.

[17] A. Moudafi, A note on the split common fixed point problem for quasi-nonexpansive operators, Nonlinear Anal. 74 (2011), 4083-4087.

[18] X. Qin, J.C. Yao, A viscosity iterative method for a split feasibility problem, J. Nonlinear Convex Anal. 20 (2019), 1497-1506. 
[19] X. Qin, A. Petrusel, J.C. Yao, CQ iterative algorithms for fixed points of nonexpansive mappings and split feasibility problems in Hilbert spaces, J. Nonlinear Convex Anal. 19 (2018), 157-165.

[20] Y.C. Tang, J.G. Peng, L.W. Liu, A cyclic algorithm for the split common fixed point problem of demicontractive mappings in Hilbert Spaces, Math. Model. Anal. 17 (2012), 457-466.

[21] H.K. Xu, A variable Krasnosel'ski $\breve{i}-M a n n$ algorithm and the multiple-set split feasibility problem, Inverse Probl. 22 (2006), 2021-2034.

[22] H.K. Xu, Iterative methods for the split feasibility problem in infinite-dimensional Hilbert spaces, Inverse Probl. 26 (2010), 105018.

[23] J. Zhao, H. Zong, Iterative algorithms for solving the split feasibility problem in Hilbert spaces, J. Fixed Point Theory Appl. 20 (2018), 11.

[24] J. Zhao, D. Hou, A self-adaptive iterative algorithm for the split common fixed point problems, Numer. Algo. 82 (2018), 1047-1063.

[25] J. Zhao, S. He, Viscosity approximation methods for split common fixed-point problem of directed operators, Numer. Funct. Anal. Optim. 36 (2015), 528-547. 